\title{
Comparing Working Memory in Old World Monkeys and Humans
}

\author{
Barry J. Richmond
}

\begin{abstract}
There are two types of neural phenomena that have been called working memory. The first is a selective, attention demanding process. In this first process, until the attention is interrupted, the memory trace has high fidelity over time. The second is a non-selective, non-attention demanding process where all events seem to form a memory traces each decaying with time. The first process is generally thought of as true working memory in humans. We started out to study the substrates of these working memory types in monkeys. To our surprise (frustration?) we found that monkeys rely primarily on the second type of working memory even when put in situations where it seems most efficacious to use the first type. The monkeys make large numbers of false positive responses related to the time in the past when a visual stimulus was presented in a sequential string of visual distractors. By way of comparison, humans heavily favor the first type of working memory; they make almost no false positive responses when asked to remember a single index stimulus. I will review our results and show how selective damage to different parts of the monkey brain thought important for supporting normal working memory function, prefrontal cortex, hippocampus, and different parts of lateral inferior temporal cortex, selectively interfere with different aspects of working memory. For physiologists this raises a problem: how do we study mechanisms of working memory using monkeys if monkeys use different strategies to solve working memory tasks?
\end{abstract}

\section{References}

Wittig, J. H., Jr., Morgan, B., Masseau, E., \& Richmond, B. J. (2016). Humans and monkeys use different strategies to solve the same short-term memory tasks. Learning and Memory, 23(11), 644-647. https://doi.org/10.1101/lm.041764.116. PMID:27918285

\section{B. J. Richmond ( $\varangle)$}

Chief Section on Neural Coding and Computation, NIMH/NIH Bldg 49,

Rm 1B-80, Bethesda, MD 20892, USA

e-mail: Barry.richmond@nih.gov 